\title{
Prevalence and Distribution of Cracked Posterior Teeth among Adult Patients
}

\author{
Temiloluwa Olawale Ogundare ${ }^{1}$, Deborah Mojirade Ajayi², Paul Ikhodaro Idon ${ }^{3}$, \\ Cornelius Tokunbo Bamise ${ }^{4}$, Adeleke Oke Oginni ${ }^{4}$, Temitope Ayodeji Esan ${ }^{4}$
}

\footnotetext{
${ }^{1}$ Department of Restorative Dentistry, Obafemi Awolowo University Teaching Hospital Complex, Ile-Ife, Nigeria

${ }^{2}$ Department of Restorative Dentistry, Faculty of Dentistry, University of Ibadan, Ibadan, Nigeria

${ }^{3}$ Department of Dental Surgery, University of Maiduguri Teaching Hospital, Maiduguri, Nigeria

${ }^{4}$ Department of Restorative Dentistry, Faculty of Dentistry, Obafemi Awolowo University, Ile-Ife, Nigeria

Email: likkysmart@yahoo.com
}

How to cite this paper: Ogundare, T.O. Ajayi, D.M., Idon, P.I., Bamise, C.T., Oginni, A.O. and Esan, T.A. (2020) Prevalence and Distribution of Cracked Posterior Teeth among Adult Patients. Open Journal of Stomatology, 10, 74-86.

https://doi.org/10.4236/ojst.2020.105009

Received: March 24, 2020

Accepted: May 6, 2020

Published: May 9, 2020

Copyright (C) 2020 by author(s) and Scientific Research Publishing Inc. This work is licensed under the Creative Commons Attribution International License (CC BY 4.0).

http://creativecommons.org/licenses/by/4.0/

\begin{abstract}
Background: There are challenges in detection and prevalence of cracked teeth, especially in asymptomatic cases. This underestimates the actual prevalence and possibility of preventive restorative treatment. Objectives: To determine the prevalence and distribution of cracked posterior teeth among adult patients attending the dental clinic of OAUTHC, Ile-Ife, Osun State, Nigeria. Materials and Methods: A cross sectional study where consecutive patients aged 18 years and above were examined for the presence of cracked posterior teeth over a period of one year (May 2016-April 2017). The teeth were initially examined with the naked eye. Thereafter, transillumination, methylene blue dye and magnifying lens were used to aid detection of more cracks. Data was analyzed with the IBM Statistical Package for Social Sciences (SPSS) for Windows (version 21.0). Level of significance was set at $\mathrm{p} \leq 0.05$. Results: Out of the 64,370 posterior teeth examined for cracks in 3345 patients, 264 were diagnosed as cracked in 152 patients, giving a patient prevalence of $4.5 \%$ and tooth prevalence of $0.4 \%$. Amongst the 152 patients, the prevalence was $9.1 \%$. There was a slightly higher male preponderance while cracked tooth was observed to increase with age $(\mathrm{p}=0.007)$. The skilled $(56.6 \%)$ and those that attained tertiary education $(77.6 \%, \mathrm{p}=0.023)$ had more cracked teeth. Mandibular first molar (19.7\%) was the most frequently cracked tooth type. The cracks were usually centrally placed (91.7\%) and run in a mesiodistal direction (61\%). Transillumination (44.7\%) detected more cracked teeth. Conclusion: Prevalence of cracked tooth was observed to be low with a slightly higher proportion in male and the figure increases with age. Mandibular first molar was the most frequently cracked tooth while transillumination was the most useful diagnostic tool. Most cracks ran in the mesiodistal direction and fracture lines were usually centrally placed.
\end{abstract}




\section{Keywords}

Cracked Tooth, Prevalence, Distribution, Adult, Posterior Tooth

\section{Introduction}

There have been challenges as regards definition, diagnosis and treatment of cracked teeth. These have made it a research problem in the literature. Earliest definition in the literature was by Gibbs [1] in 1954. He referred to the clinical symptoms associated with incomplete fracture of posterior teeth involving the cusp as "cuspal fracture odontalgia". The American Association of Endodontists [2] defined a cracked tooth as a type of incomplete longitudinal tooth fracture usually involving the premolars and molars. The fracture plane usually originates from the coronal aspect of the tooth and progresses apically with or without symptoms depending on the extent of fracture [2]. Other longitudinal fractures described by this association include crazed tooth, fractured cusp, vertical root fracture and split tooth. Apart from crazed tooth, the others could result from an untreated cracked tooth. Some authors [3] [4] [5] also use the term "cracked tooth syndrome" to describe this type of longitudinal tooth fracture with associated signs and symptoms.

Guertsen [5] in 2003, reported that the prevalence of cracked tooth had been on the rise over the past decade. Furthermore, splits, cracks or fractures have been reported to be the third most common cause of tooth loss in industrialized countries in recent times [3]. This, coupled with variations among different populations and the increasing rate shows it is of high clinical importance. Bader et al. [6] reported that longitudinal tooth fractures such as cracked tooth, fractured cusp, vertical root fracture and split tooth accounted for a rate of 4.4 fractured teeth per 100 adults per year for posterior teeth, with $15 \%$ of such fractures resulting in pulpal involvement or extraction. They further reported the corresponding rates for molars and premolars as 3.1 and 1.3 teeth per 100 adults respectively, from the total number of fractures seen in the posterior teeth. Cracked tooth usually presents in patients aged between 30 and 50 years [7] [8] [9]. Predominance in either of the genders has been reported [10] [11], while others have reported no sex predilection [12] [13]. Most studies [10] [11] [14] have reported cracked teeth to be most prevalent in mandibular molars with intracoronal restorations.

As regards the direction of crack lines, most cracks usually run in the mesiodistal direction; however, they may run in the buccolingual direction or as a combination of both [9] [12]. Crack lines are mostly centrally located [11] [15]. Centrally located cracks and those running in a mesiodistal direction are more likely to cause pulpal involvement, while peripherally located cracks more often lead to cuspal fracture [9]. Cracks in teeth tend to follow these areas because they are weak portions of the tooth where fracture plane may likely propagate [3]. 
Variations in the reported prevalence may be affected not only by population characteristics, but also importantly by the challenge in identifying the cracks, especially in asymptomatic teeth. Cracked teeth may or may not be symptomatic. When present, symptoms are non-specific and mimic pulpal, periapical or periodontal inflammation. The detection of the un-displaced crack line is challenging in clinical practice [2] [3] [10], and more so in asymptomatic cracked teeth. In the presence of symptoms, the clinician will usually attempt to seek for the cause of pain, and the presence of cracks eventually becomes obvious. Identifying cracked teeth in the absence of symptoms, however, poses a different challenge. The clinician may naturally not seek for cracks during routine oral examinations, and individuals will usually not present to the clinic due to cracked teeth without symptoms. They may, therefore, be found by chance when patients report for other complaints. Such incidental findings would be more probable when they are visible to the naked eye. Otherwise, they would go unnoticed and continue to propagate as a result of mastication or certain parafunctional habits. However, with the use of other diagnostic aids, more cracks can be detected. In the absence of symptoms, these adjuncts, such as dyes, transillumination, and magnification become necessary. Prevalence studies on cracked teeth would, therefore, require the use of one or more of these additional methods to prevent underestimating the problem.

Studies [11] [15] [16] [17] have been done on the prevalence of cracked teeth. However, not many published studies were found on the prevalence of cracked teeth in Nigeria. Udoye and Jafarzadeh [11] conducted their study among Nigerian patients in the southeastern part of the country. There is yet no such data on the condition in other regions. Availability of such data on early and accurate diagnosis of cracked teeth provide options for conservation of such teeth as well as better prognosis. As research into improvements in the diagnosis and treatment of cracked teeth continues, this study became necessary and was, therefore, carried out with the aim of determining the prevalence and distribution of cracked teeth in adult patients presenting at the Dental Hospital of the Obafemi Awolowo University Teaching Hospitals Complex, located in the Southwest of Nigeria.

\section{Materials and Methods}

A cross sectional study was conducted over a one-year period (May 2016-April 2017) among all patients aged 18 years and above that presented at the Oral diagnosis and Restorative clinics of the teaching hospital. Approval for the study (ERC/2014/11/07) was granted by the Ethics committee of the hospital before commencement. Consecutive adult patients in good general health were examined irrespective of their primary reason for visit to the facility, following explanation of the study and their consent to participate.

The minimum sample size required for the study was based on recruiting patients consecutively for a period of one year while using the formula below [18]. 


$$
N=\frac{Z^{2} p(1-p)}{D^{2}}
$$

where $N$-is the total sample size, $Z$ is taken as 1.96 at $95 \%$ confidence interval, $P($ prevalence $)=0.089$, as obtained from a study with similar methodology [11] and $D$ (degree of accuracy) $=0.05$.

Based on the above formula, $\mathrm{N}$ was calculated to be $=124.59 \approx 125$.

To accommodate for attrition, $21.6 \%$ of sample size (27 subjects) was added to the value obtained (125 subjects); resulting in a sample size of 152 consecutive subjects for the study.

Intraoral examination with a sterile dental probe and mirror was carried out to detect presence of clinically visible cracked posterior tooth or teeth. The diagnostic criteria for the cracked tooth were: a posterior tooth with an identifiable crack line or fracture running in a mesiodistal or buccolingual direction or both; crack line that is inseparable by a dental probe; and the tooth may be either symptomatic or asymptomatic. Adjuncts such as magnification lens, transillumination and staining were used to aid the identification of cracked tooth.

Initially, each posterior tooth was examined for cracks with the naked eye without any adjunct. Afterwards the magnification lens was utilized with a 3.5X (14 diopter) power magnification lens (SE Japan). Transillumination using Microlux transilluminator (Microlux Diagnostic System) was subsequently done to lighten up the tooth surface properly. The transilluminator was also placed against the buccal aspect of the tooth while a dental mirror placed on the lingual aspect was checked if light passes through the tooth or not. The methylene blue was used on the teeth which were symptomatic, yet, no visible crack line was detected. The teeth were stained for a minute and rinsed afterwards to assess if suspected crack lines retained the dye. For each cracked posterior tooth detected through the naked eye or with the adjuncts, the dental probe was passed gently along the crack to rule out a split tooth.

Following confirmation, patients with at least one cracked posterior tooth were recruited into the study. Anterior teeth with cracks and other longitudinal fractures such as craze lines, fractured cusps, vertical root fracture and split tooth were excluded from the study. All study related data including subjects' characteristics were collected in a data form including age, gender, occupation, education, tooth involved, number of teeth involved and distribution.

Data analysis: Data was analyzed with the IBM Statistical Package for Social Sciences (SPSS) for Windows (version 21.0). Associations between sociodemographic variables and prevalence of cracked teeth; direction, location of cracks and diagnostic aids were carried out with Chi square test at $95 \%$ confidence interval, with tables and charts utilized for data presentation. The level of statistical significance was set at $\mathrm{p}$-value $\leq 0.05$.

\section{Results}

A total of 3345 patients, comprising 1642 males and 1703 females, with ages 
ranging from 18 to 92 years and a mean age of $54.26 \pm 16.54$ years presented at the oral diagnosis clinic during the 12-month study period. One hundred and fifty-two (152) of these patients were identified with varying numbers of cracked posterior teeth, which gave an overall prevalence rate of $4.54 \%$. The highest prevalence was seen among subjects in the 41 - 50-year age range and those above 60 years $(1.17 \%$ each) with the least found among those less than 20 years $(0.06 \%)$. Prevalence was observed to increase with age and found to be significant $(\mathrm{p}<0.05)$. The difference in prevalence between the genders was not statistically significant, though commoner among the males (Table 1 ).

From a total of 64,370 posterior teeth examined in the 3345 subjects, 264 posterior teeth were diagnosed cracked. This gave a tooth prevalence of $0.4 \%$. The 264 cracked posterior teeth were identified among the 2905 posterior teeth examined in the 152 subjects, giving a cracked posterior teeth prevalence of $9.1 \%$ among subjects identified with cracked posterior teeth. Their ages ranged from 18 - 84 years with a mean age of $50.43 \pm 14.18$ years. Those in the $51-60$ years age range and those above 60 years had the highest prevalence of cracked teeth with the least among those aged 20 years or less. Distribution of the cracked teeth based on other subjects' variables-gender, education and occupation showed differences but statistically significant only for level of education ( $\mathrm{p}=$ $0.023)$. Greater in males $(p=0.987)$, skilled subjects $(p=0.542)$ and those with tertiary education (Table 2 ).

The number of cracked teeth ranged from 1 to 5 teeth per subject, with one cracked tooth each identified in $96(63.2 \%)$ subjects. The maximum number of cracked teeth (5) was noted in $4.6 \%$ of the subjects (Table 3 ).

The most observed cracked tooth was the mandibular first molar (19.7\%), followed by the mandibular first premolar (16.7\%), then the mandibular second molar (15.2\%) while the least was the maxillary third molar accounting for $1.5 \%$ of the total cracked teeth (Figure 1).

Using a combination of different methods to identify the presence of cracks in the posterior teeth, only 88 (33.3\%) were visible to the naked eye alone, while the use of magnification lens, transillumination, and staining were necessary for identification of the rest of the cracked teeth (66.7\%) (Figure 2).

The majority (91.7\%) of the cracks were located centrally on the teeth while most (61.0\%) of the cracks ran in a mesiodistal direction (Table 4).

Transillumination (41.7\%) was the most useful in detecting centrally located cracks. This was followed by the naked eye (36.0\%) while the use of dye was the least $(9.9 \%)$. As regards the peripherally located cracks, transillumination was also found to detect the majority $(77.3 \%)$ while the use of dye and magnification accounted for $9.1 \%$ each with naked eye being the least (4.5\%). The relationship between the diagnostic tools and location of cracks were all found to be statistically significant (Table 5).

Majority (65.2\%) of the cracks running in the mesiodistal direction were detected with transillumination, followed by magnification (18.6\%), use of dye (12.4\%) and naked eye being the least (3.7\%). Naked eye detected most (82.8\%) 
of the cracks running buccolingually, followed by transillumination (13.1\%), use of dye (6.1\%) and magnification (2.0\%). All the combined cracks were detected with the naked eye. The relationship between the diagnostic tools and direction of cracks were all statistically significant except the use of dye (Table 6).

Table 1. Prevalence of cracked teeth among the subjects by age group and gender.

\begin{tabular}{|c|c|c|c|}
\hline Age group & Present & Absent & Total \\
\hline & n (\%) & n (\%) & $\mathrm{n}(\%)$ \\
\hline$\leq 20$ & $2(0.06)$ & 397 (11.87) & 399 (11.93) \\
\hline $21-30$ & $13(0.39)$ & $1006(30.07)$ & $1019(30.46)$ \\
\hline $31-40$ & $21(0.63)$ & $538(16.08)$ & $559(16.71)$ \\
\hline $41-50$ & 39 (1.17) & $362(10.82)$ & 401 (11.99) \\
\hline $51-60$ & $38(1.14)$ & $487(14.56)$ & $525(15.70)$ \\
\hline$>60$ & 39 (1.17) & $493(14.74)$ & $532(15.91)$ \\
\hline Total & $152(4.54)$ & $3193(95.46)$ & $3345(100.0)$ \\
\hline \multicolumn{4}{|l|}{ Gender } \\
\hline Male & $79(2.36)$ & $1563(46.73)$ & $1642(49.01)$ \\
\hline Female & $73(2.18)$ & $1630(48.73)$ & 1703 (50.91) \\
\hline Total & $152(4.54)$ & $3193(95.46)$ & $3345(100.0)$ \\
\hline
\end{tabular}

Chi-square tests: (Age $\left.X^{2}=21.461, \mathrm{df}=5, \mathrm{p}=0.007\right),\left(\right.$ Gender $\left.X^{2}=2.907, \mathrm{df}=1, \mathrm{p}=0.100\right)$.

Table 2. Comparison of the number of cracked teeth by subjects' sociodemographic characteristics.

\begin{tabular}{|c|c|c|c|c|c|}
\hline Parameters & & $\begin{array}{c}\mathrm{N}_{\mathrm{p}}=152 \\
\mathrm{n}(\%)\end{array}$ & $\begin{array}{c}\mathrm{N}_{\mathrm{T}}=264 \\
\mathrm{n}(\%)\end{array}$ & $x^{2}$ & $p$ value \\
\hline \multirow{6}{*}{$\begin{array}{l}\text { Age group } \\
\text { (years) }\end{array}$} & $\leq 20$ & $2(1.3)$ & $2(0.8)$ & \multirow{6}{*}{21.889} & \multirow{6}{*}{$0.009^{\star *}$} \\
\hline & $21-30$ & $13(8.6)$ & $15(5.7)$ & & \\
\hline & $31-40$ & $21(13.8)$ & $27(10.2)$ & & \\
\hline & $41-50$ & $39(25.7)$ & $65(24.6)$ & & \\
\hline & $51-60$ & $38(25.0)$ & $78(29.5)$ & & \\
\hline & $>60$ & $39(25.7)$ & 77 (29.2) & & \\
\hline \multirow[b]{2}{*}{ Sex } & Male & $79(52.0)$ & $139(52.7)$ & \multirow[b]{2}{*}{3.001} & \multirow[b]{2}{*}{0.987} \\
\hline & Female & $73(48.0)$ & $125(47.3)$ & & \\
\hline \multirow{3}{*}{ Occupation } & Skilled & $86(56.6)$ & $154(58.3)$ & \multirow{3}{*}{2.891} & \multirow{3}{*}{0.542} \\
\hline & Unskilled & $34(22.4)$ & $57(21.6)$ & & \\
\hline & Dependants & $32(21.1)$ & $53(20.1)$ & & \\
\hline \multirow{4}{*}{ Education } & Nil & $5(3.3)$ & $14(5.3)$ & \multirow{4}{*}{14.003} & \multirow{4}{*}{$0.023^{\star *}$} \\
\hline & Primary & $6(3.9)$ & $13(4.9)$ & & \\
\hline & Secondary & $23(15.1)$ & $49(18.6)$ & & \\
\hline & Tertiary & $118(77.6)$ & $188(71.2)$ & & \\
\hline
\end{tabular}

$\mathbf{N}_{\mathrm{p}}=$ total number of patients; $\mathbf{N}_{\mathrm{T}}=$ total number of cracked teeth. 
Table 3. Number of cracked posterior teeth per patient.

\begin{tabular}{ccc}
\hline $\begin{array}{c}\text { Number of cracked } \\
\text { teeth per subject }\end{array}$ & $\begin{array}{c}\text { Number of patients } \\
\mathbf{N}_{\mathrm{p}}(\%)\end{array}$ & $\begin{array}{c}\text { Cumulative number } \\
\text { of cracked teeth } \mathrm{N}_{\mathrm{T}}(\%)\end{array}$ \\
\hline 1 & $96(63.2)$ & $96(36.4)$ \\
2 & $23(15.1)$ & $46(17.4)$ \\
3 & $17(11.2)$ & $51(19.3)$ \\
4 & $9(5.9)$ & $36(13.6)$ \\
5 & $7(4.6)$ & $35(13.3)$ \\
Total & $152(100)$ & $264(100)$ \\
\hline
\end{tabular}

$\mathbf{N}_{\mathrm{p}}=$ total number of patients; $\mathbf{N}_{\mathrm{T}}=$ total number of cracked teeth.

Table 4. Direction and location of cracks.

\begin{tabular}{lcc}
\hline & Classification & $\mathbf{N}_{\mathrm{T}}=264$ \\
\hline \multirow{2}{*}{ Location of cracks } & Central & $242(91.7)$ \\
& Peripheral & $22(8.3)$ \\
& Mesiodistal & $161(61.0)$ \\
Direction of cracks & Buccolingual & $99(37.5)$ \\
& Combination & $4(1.5)$ \\
\hline
\end{tabular}

Table 5. Relationship between location of cracks and diagnostic tools.

\begin{tabular}{cccccc}
\hline Diagnostic aids & \multicolumn{2}{c}{ Location of cracks } & Total & $\mathbf{x}^{2}$ & p value \\
\hline & $\begin{array}{c}\text { Central } \\
\mathbf{n}=\mathbf{2 4 2} \\
\mathbf{n}(\%)\end{array}$ & $\begin{array}{c}\text { Peripheral } \\
\mathbf{n}=22 \\
\mathbf{n}(\%)\end{array}$ & & & \\
& $87(36.0)$ & $1(4.5)$ & 88 & 54.096 & $<0.001$ \\
Naked eye & $101(41.7)$ & $17(77.3)$ & 118 & 74.336 & $<0.001$ \\
Transillumination & $24(9.9)$ & $2(9.1)$ & 26 & 14.226 & 0.001 \\
Dye & $30(12.4)$ & $2(9.1)$ & 32 & 22.718 & $<0.001$ \\
\hline Magnification & & & & & \\
\hline
\end{tabular}

Table 6. Relationship between direction of cracks and diagnostic tools.

\begin{tabular}{ccccccc}
\hline Diagnostic aids & \multicolumn{3}{c}{ Direction of cracks } & Total & $\mathbf{x}^{2}$ & p value \\
\hline & $\begin{array}{c}\text { Mesiodistal } \\
\mathbf{n = 1 6 1} \\
\mathbf{n}(\%)\end{array}$ & $\begin{array}{c}\text { Buccolingual } \\
\mathbf{n = 9 9} \\
\mathbf{n}(\%)\end{array}$ & $\begin{array}{c}\text { Combination } \\
\mathbf{n}=\mathbf{4} \\
\mathbf{n}(\%)\end{array}$ & & & \\
Naked eye & $6(3.7)$ & $82(82.8)$ & $4(100)$ & 88 & 200.997 & $<0.001$ \\
Transillumination & $105(65.2)$ & $13(13.1)$ & $0(0.0)$ & 118 & 70.565 & $<0.001$ \\
Dye & $20(12.4)$ & $6(6.1)$ & $0(0.0)$ & 26 & 3.238 & 0.198 \\
Magnification & $30(18.6)$ & $2(2.0)$ & $0(0.0)$ & 32 & 20.602 & $<0.001$ \\
\hline
\end{tabular}




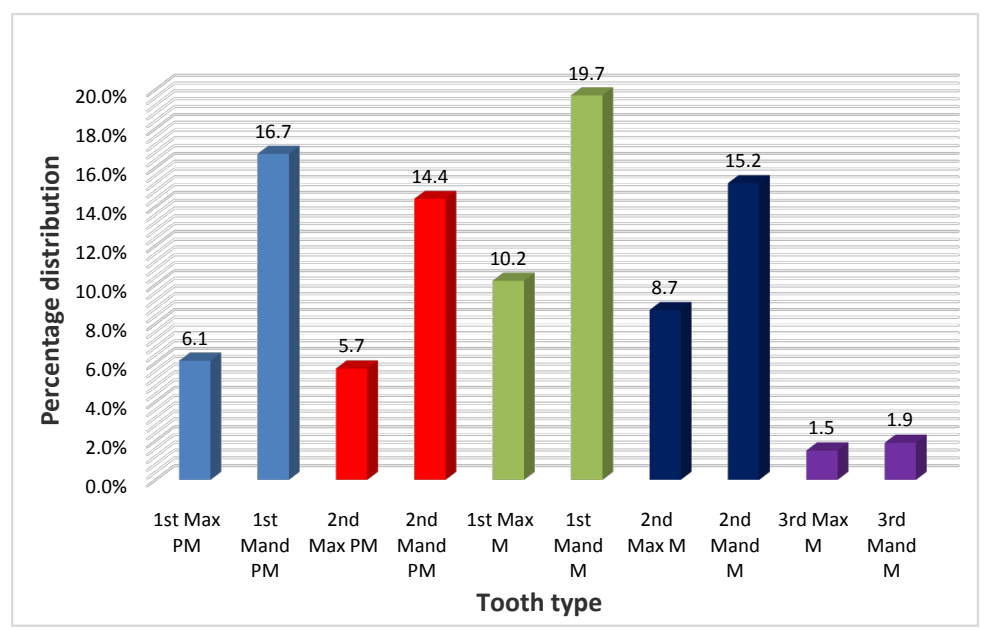

Figure 1. Distribution of cracked tooth according to tooth types. MaxMaxillary, Mand-Mandibular, PM-Premolar, M-Molar.

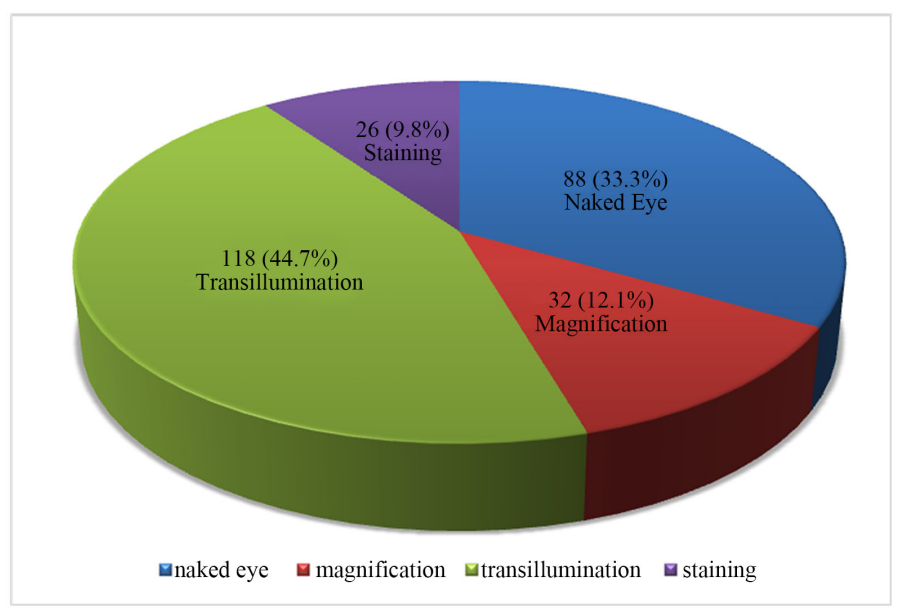

Figure 2. Diagnostic tools used in identifying cracked teeth.

\section{Discussion}

Cracked teeth may occur with or without symptoms, and as a finding, even in the presence of pulpal symptoms, may be challenging to locate. Problems have been associated with the identification of cracked teeth as crack detection may require thorough assessment [2]. Seo [19] reported the importance of using different diagnostic tools and aids in identifying cracks in teeth. In this study, the detection of cracked teeth was enhanced by the use of various diagnostic tests to avoid missing out the less visible cracks, a factor that may underestimate the prevalence. Transillumination was most useful accounting for nearly half of the detected cracked teeth. Apart from the subtle nature of the cracks that necessitated the use of this adjunct, another reason may be due to the usual mesio-distal direction of the majority of cracks [13] as was also observed in this study, a factor that makes transillumination a good tool for its detection. The light beam applied to the buccal or lingual surface of the tooth may not completely pass through to the other side of the tooth, indicating a longitudinal tooth fracture 
with crack line in a mesiodistal direction. The usefulness of this method was also affirmed in this study. The number of mesiodistal cracks detected with transillumination was very high (65.2\%) when compared to buccolingual cracks and combined cracks that were detected usually on visual examination without transillumination.

Furthermore, the centrally placed cracks were easily detected when compared to the peripherally located cracks which required more of transillumination in its detection. This may be due to the usual complaint of pain from teeth with centrally located cracks following pulpal involvement which makes the examiner look for cracks in such teeth. Also, peripherally located cracks like cracks running in the mesiodistal direction may be difficult to detect because of the adjacent teeth.

Staining was the least useful in the detection of cracked teeth in this study. This is probably because crack detection by staining requires the stain to be in close contact with the tooth for two to three days. Since detection is not immediate, this gives other methods like transillumination, higher usage and thus a higher rate of crack detection. However, it is a useful tool when it comes to delineating crack lines.

In spite of the diagnostic aids used, the prevalence observed in this population (4.5\%) was lower than that reported in most studies [11] [16] [17]. For example, Udoye and Jafarzadeh [11] reported a prevalence of $8.9 \%$ in South Eastern Nigeria. Krell and Rivera [17] reported a prevalence of 9.7\% in an American population while Detar [16] in another group of American population reported a slightly higher prevalence of 5.9\%. Differences in diet, life expectancy, accessibility to and utilization of dental services as well as the use of advanced technology in diagnosing cracked teeth could cause the variations in the results. This study observed a very low tooth prevalence $(0.4 \%)$ of cracks among the posterior teeth examined in the whole population of subjects examined. None of the studies found in the literature reported tooth prevalence of cracks among all posterior teeth examined, making it difficult to make comparison. However, like in this study, Siribang and Sirikwan [15] reported cracked posterior tooth prevalence among subjects with cracked teeth. A lower value of $9.1 \%$ prevalence among the posterior teeth examined in subjects with cracked teeth was observed in this study, compared to $37.9 \%$ reported by Siribang and Sirikwan [15]. Aside from differences in population characteristics and diagnostic methods used, the difference in prevalence could also have been due to the inclusion of craze lines in their study. Majority (63.2\%) of the subjects in this study had at least one cracked tooth. Similarly, majority (97\%) of patients studied by Siribang and $\mathrm{Si}$ rikwan [15] had at least one cracked tooth. The presence of more than one cracked tooth in some of the subjects seen agrees with the study of Cameron [4] that reported that it is not uncommon to find more than one cracked tooth in an individual at a given time. He stated that the predisposing factors of cracked tooth usually work in concert on multiple teeth at the same time. 
Generally, the prevalence of cracked tooth increases with age [2] [11] [12] [20]. This study also found the prevalence of cracked tooth to increase with age. The reason adduced to explain this observation was the retention of teeth till old age which makes them undergo repeated occlusal and thermal stresses over a long period of time. This may cause dehydration and fatigue with subsequent loss of resistance of human dentine to crack formation [21] [22].

Different prevalence scores of cracked teeth by sex have been reported [2] [11] [12] [15] [16]. Udoye and Jafarzadeh [11] as well as other studies found males to be more affected. Helkimo and Ingerwall [23] suggested that masticatory muscles are more developed in males than females making them exert more masticatory force which may predispose the teeth to cracks. On the contrary, other studies [2] [15] [16] [24] have reported a higher female predilection. Cameron [10] stated that this may be due to better accessibility and utilization of dental services by the females. The present study found a slightly higher prevalence in males, though not statistically significant. This is in agreement with the report of Dewberry [25] that also found a little higher number of cracked teeth in males. The varying predilections reported for the genders may be due to differences in the population base, gender composition and attendance in the dental clinics where the studies were carried out.

The education level of the subjects was skewed in number towards subjects with tertiary education. This is probably due to the location of the dental hospital in an academic environment, a university. Therefore, the majority of the patients could have been students and workers at the University. The higher number of cracked teeth observed in subjects that attained tertiary education is thus expected. Furthermore, the educated and higher socioeconomic class tend to take more interest in their health, and are also able to afford treatment.

Most studies have demonstrated cracked tooth to occur more in the mandible than the maxilla [9] [10] [26] [27]. The reasons are that the posterior mandibular teeth lack structural integrity and are subjected to heavier masticatory forces [10]. This study noted that more mandibular posterior teeth were affected with cracks. In contrast, some studies [11] [13] demonstrated more cracked teeth in the maxilla. Roh and Lee [13] observed more cracked teeth in the maxilla of Koreans and suggested that the presence of lingually inclined buccal cusp of mandibular molars that acts as a plunger cusp on the maxillary molars in that population may account for this occurrence. Cracked tooth has been demonstrated to affect molars more than other tooth types [11] [13] [16] [19]. Similarly, the same trend was observed in this study. Ehrmann and Tyas [26] suggested the cause to be the proximity of molars to the temporomandibular joint. Arnold [28] reported that heavier forces are exerted on most posterior teeth with a force ratio of 4:2:1 on molars, premolars, and incisors respectively.

This study further found the mandibular first molar to be the most affected tooth type as reported by previous studies [14] [29]. This is not unexpected because it is the first tooth to erupt. It stays longer and undergoes lots of occlusal 
stress compared to other posterior teeth. Furthermore, they could be more susceptible to dental caries, masticatory accident, repeated restorations and occlusal wear facets. Thus, it could be stated that the synergistic effect of these factors on the mandibular first molar predispose them to have more cracks. On the contrary, other studies [10] [11] reported the mandibular second molar to be the most commonly cracked tooth.

The limited diagnostic methods employed in this study, like others before this, could be a limitation to reporting the true prevalence of cracked teeth. Some authors [30] [31] have reported increased ability of new technology to detect presence of more cracks in teeth over the well-known established ones like transillumination, staining with dyes, and magnification with loupes. These include operating microscopes [30] [31] that can give up to $16 \mathrm{X}$ magnification of the tooth structure and ultrasound [32]. Also, with the advent of cone beam computed tomography (CBCT) and optical coherence tomography, it is expected that there would be improvement in locating cracks usually not visible to the naked eyes, most especially those located in the roots. Although, these facilities are expensive and not readily available in most centers, they are important diagnostic aids that could enhance the detection of more cracked teeth.

\section{Conclusion}

The study found a low prevalence of $4.5 \%$ among the dental patients of the hospital, a $0.4 \%$ posterior tooth prevalence, a slightly higher prevalence in males, and a prevalence that increased with age. The majority of cracks were found in mandibular molars and the mandibular first molar was the most frequently cracked tooth. Transillumination is a quick and easy way to enhance the detection of cracked teeth, but a combination of other methods provides better assessment.

\section{Conflicts of Interest}

The authors declare no conflicts of interest regarding the publication of this paper.

\section{References}

[1] Gibbs, J.W. (1954) Cuspal Fracture Odontalgia. Dent Digest, 60, 158-160.

[2] American Association of Endodontists (2008) Endodontics: Colleagues for Excellence. Cracking the Cracked Tooth Code: Detection and Treatment of Various Longitudinal Tooth Fractures. AAE, 1-13.

[3] Lynch, C.D. and McConnell, R.J. (2002) The Cracked Tooth Syndrome. Journal of the Canadian Dental Association, 68, 470-475.

[4] Cameron, C.E. (1964) Cracked Tooth Syndrome. Journal of the Canadian Dental Association, 68, 405-411. https://doi.org/10.14219/jada.archive.1964.0108

[5] Guertsen, W. (2003) Diagnosis, Therapy, and Prevention of the Cracked Tooth Syndrome. Quintessence International, 34, 409-417.

[6] Bader, J.D, Martin, J.A. and Shugars, D.A. (1995) Preliminary Estimates of the In- 
cidence and Consequences of Tooth Fracture. Journal of the Canadian Dental Association, 126, 1650-1654. https://doi.org/10.14219/jada.archive.1995.0113

[7] Ellis, S.G. (2001) Incomplete Tooth Fracture: Proposal for a New Definition. British Dental Journal, 190, 424-428. https://doi.org/10.1038/sj.bdj.4800992

[8] Synder, D.E . (1976) The Cracked Tooth Syndrome and Fractured Posterior Cusp. Oral Surgery, Oral Medicine, Oral Pathology, 41, 698-704. https://doi.org/10.1016/0030-4220(76)90181-X

[9] Hiatt, W.H. (1973) Incomplete Crown-Root Fracture in Pulpal-Periodontal Disease. Journal of Periodontology, 44, 369-379. https://doi.org/10.1902/jop.1973.44.6.369

[10] Cameron, C.E. (1976) The Cracked Tooth Syndrome: Additional Findings. The Journal of the American Dental Association, 93, 971-975. https://doi.org/10.14219/jada.archive.1976.0034

[11] Udoye, C.I. and Jafarzadeh, H. (2009) Cracked Tooth Syndrome: Characteristics and Distribution among Adults in a Nigerian Teaching Hospital. Journal of Endodontics, 35, 34-36. https://doi.org/10.1016/j.joen.2008.12.011

[12] Turp, J.C. and Gobetti, J.P. (1996) The Cracked Tooth Syndrome: An Elusive Diagnosis. The Journal of the American Dental Association, 127, 1502-1507. https://doi.org/10.14219/jada.archive.1996.0060

[13] Roh, B.D. and Lee, Y.E. (2006) Analysis of 154 Cases of Teeth with Cracks. Dental Traumatology, 22, 118-123. https://doi.org/10.1111/j.1600-9657.2006.00347.x

[14] Abou-Rass, M. (1983) Crack Lines: The Precursors of Tooth Fractures; Their Diagnosis and Treatment. Quintessence International, 14, 437-447.

[15] Siribang-on, P.K. and Sirikwan, S. (2014) Prevalence of Cracked Tooth in a Group of Patients at the Faculty of Dentistry, Mahidol University. Mahidol Dental Journal, 34, 234-242.

[16] Detar, M.S. (2014) Evaluation of the Prevalence and Clinical Characteristics of Intrapulpal Cracks Utilizing a Novel Classification System. Virginia Commonwealth University, Richmond, VA.

[17] Krell, K.V. and Rivera, E.M. (2007) A Sixteen Year Evaluation of Cracked Teeth Diagnosed with Reversible Pulpitis: Treatment and Prognosis. Journal of Endodontics, 33, 1405-1407. https://doi.org/10.1016/j.joen.2007.08.015

[18] Araoye, M.O. (2003) Subject Selection in Research Methodology with Statistics for Health and Social Sciences. Nathadex, Ilorin, 115-129.

[19] Seo, D.G. (2012) Analysis of Factors Associated with Cracked Teeth. Journal of Endodontics, 38, 288-292. https://doi.org/10.1016/j.joen.2011.11.017

[20] Ellis, S.G., Macfarlane, T.V. and Mc Cord, J.F. (1999) Influence of Patient Age on the Nature of Tooth Fracture. Journal of Prosthodontics, 82, 226-230. https://doi.org/10.1016/S0022-3913(99)70161-7

[21] Bajaj, D., Sundaram, N., Nazari, A. and Arola, D. (2006) Age, Dehydration and Fatigue Crack Growth in Dentine. Biomaterials, 27, 2507-2517. https://doi.org/10.1016/j.biomaterials.2005.11.035

[22] Eakle, W.S., Maxwell, E.H. and Braly, B.V. (1986) Fractures of Posterior Teeth in Adults. The Journal of the American Dental Association, 112, 215-218. https://doi.org/10.14219/jada.archive.1986.0344

[23] Helkimo, E. and Ingerwall, B. (1978) Bite Force and Functional State of the Masticatory System in Young Men. Swedish Dental Journal, 2, 167-175.

[24] Homewood, C.I. (1998) Cracked Tooth Syndrome-Incidence, Clinical Findings and Treatment. Australian Dental Journal, 43, 217-222. 
https://doi.org/10.1111/j.1834-7819.1998.tb00167.x

[25] Dewberry, J.A. (1996) Vertical Fractures of Posterior Teeth. In: Weine, F.S., Ed., Endodontic Therapy, 5th Edition, Mosby, St Louis, MO, 71-81.

[26] Ehrmann, E.H. and Tyas, M.T. (1990) Cracked Tooth Syndrome: Diagnosis, Treatment and Correlation between Symptoms and Post-Extraction Findings. Australian Dental Journal, 35, 105-112. https://doi.org/10.1111/j.1834-7819.1990.tb05872.x

[27] Lubisich, E.B., Hilton, T.J. and Ferracane, J. (2010) Cracked Teeth: A Review of the Literature. Journal of Esthetic and Restorative Dentistry, 22, 158-167. https://doi.org/10.1111/j.1708-8240.2010.00330.x

[28] Arnold, M. (1981) Bruxism and the Occlusion. Dental Clinics of North America, 25, 395-407.

[29] Ratcliff, S., Becker, I.M. and Quinn, L. (2001) Type and Incidence of Cracks in Posterior Teeth. Journal of Prosthetic Dentistry, 86, 168-172. https://doi.org/10.1067/mpr.2001.116578

[30] Alex, W.C. (2004) Diagnosis and Management of Cracked Teeth. Hong Kong Dental Journal, 1, 78-84.

[31] Clark, D.J., Cherilyn, G.S. and Jacinthe, M.P. (2003) Definitive Diagnosis of Early Enamel and Dentin Cracks Based on Microscopic Evaluation. Journal of Esthetic and Restorative Dentistry, 15, 391-401. https://doi.org/10.1111/j.1708-8240.2003.tb00963.x

[32] Culjat, M.O., Singh, R.S. and Brown, E.R. (2005) Ultrasound Crack Detection in a Simulated Human Tooth. Dento-Maxillo-Facial Radiology, 34, 80-85.

https://doi.org/10.1259/dmfr/12901010 